\title{
مميزات تعدد اللغات في بناء عالم
}

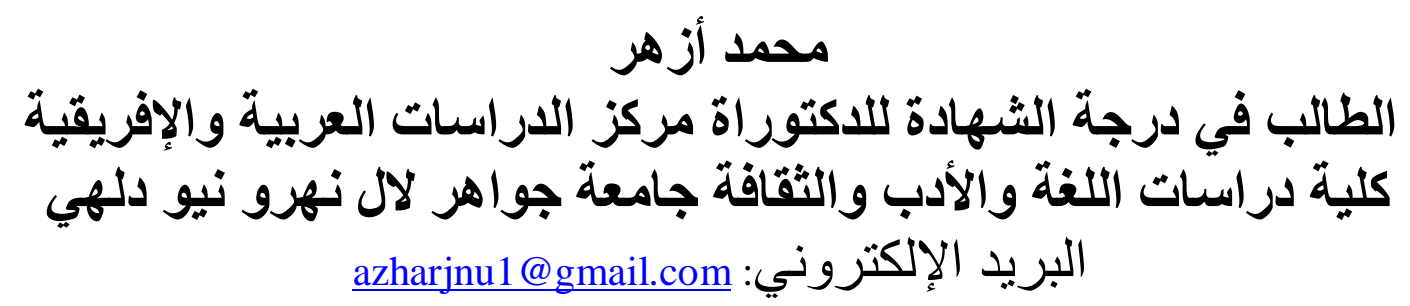

\begin{abstract}
The Advantages of Multilingualism in Building a World. This paper is aimed at describing multilingualism In India, a country known with multilingual and multicultural people. India is the most multilingual country in the world and it is the second largest country with a population of more than 17 percent of the world's population. Knowing two or more than two languages becomes the need for communication among speech communities as well as individuals. Defined as an occurrence regarding an individual speaker who uses two or more languages, multilingualism basically arises due to the need to communicate across speech communities. Multilingualism is not a rare but a normal necessity across the world due to globalization and wider cultural communication. Also it is not a recent phenomenon; it was prevalent in the ancient time also. This means that the perspective of global citizenship has achieved a remarkable achievement. There is no doubt in history that India is one of the oldest civilizations and a source of other civilizations in the world and draws the attention of researchers, and is of great importance at the global level.
\end{abstract}

Keywords: multilingualism; communication; building a world

الحياة مع الحب والفرحة. وهذا البلد

الذي يسكنه المليار و هي ثثاني أكبر دولة بعد الصين في بنسبة عدد السكان. ومن الأعجب، كلنا نعيش مع الحب والوئام رغم اختلاف اللون و الثقافة و الديانة. والهند بنفسه مثنال حي للمواطنة العالمية ومثال حي للعائلة الصغيرة و هي في الواقع مثال حي لعالم أصبح كوخا صغيرا. في كل زاويتها تجد ثقافة ولغة

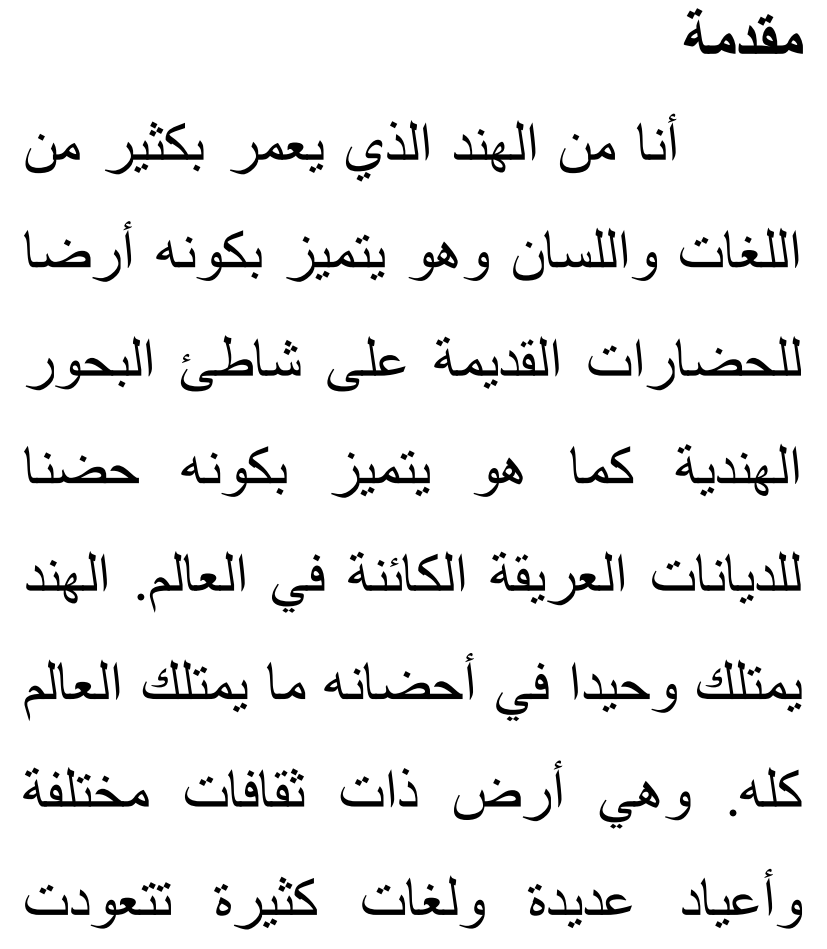


أن الهند بعتبر العالم عائلة وأسرة و Vasudhaiva"1 شعاره" العالم أسرة و احدة 2 kutumbakam ملاحمه، و هو بشمل الحبو انات و التباتات و المناخ و البيئة وسيأر الكائنات الحية. وهو بنادي للأمن اللعالمي إذ هو بقول ويقبل" إمكانبة كل دين" Sarva Panth".

Sambhav

و الرؤبة التهنية في الو في

عبارة عن السلم و عدم الثدة و العصبية، كما هي رمز انتصار الحق و الصدق ضد الخر افات و الكذب." 4 "Satyamev وjayate الهند عبارة عن أرض بعه باحتفاظ البيئة و المناخ بأنه بعطي البيئة منزلة الاله ويؤمن بعبادتها ويمنع من قطعها واستخلالها إلا أن بكون واجبا، وهو عبارة عن أرض تعطي ضبيوفها

${ }^{1}$ Robin Seelan (2015), Deconstructing Global Citizenship (Editors: Hassan Bashir and Phillips Gray), Routledge, ISBN 978-1498502580, page 143;

${ }^{2}$ Mahopanishad VI.71-73.

${ }^{3}$ Rakhit, Maanoj. RKM Propagating the Opposite of What Vivekananda and Ramakrishna Had Said: Call to the Rank and File at RKM! Stand Up and Uphold the Truth. Maanoj Rakhit. ISBN 9788189746490.

${ }^{4}$ Swami Krishnananda. "The Mundaka Upanishad:Third Mundaka, First Khanda"
ولهجة تختلف عن ثقافة ولغة ولهجة في نفس التهد.

والمجتمعات الهندية التي تتعود حياة التعايش السلمي والتي تجعل المواطنة العالمبة ممكنة. وهي يمكن أن تلعب دورا بارزا في تحقيق الأحلام المواطنة. ويوجد مفهوم المواطنة العالمية في حضنارته منذ عصر سحيق. الروئية الرهنية حول المواطنة العالمبة منذ أن كان الهند بيتا للمالئين من الخارج الذين نزلو ا الهند من كل أقاصي العالم و أدانيه واستوطنوه سكنا. فهو بيت المسلمين الذين أتوها من إيران و العرب وتركيا، وهو بيت الطائفة التي نزلت من أسيا الوسطى، ومزجت مع الهنود الأصليين و أصبحت جزء لها. و الملخص أن الهند كان يمثل لمواطنة عالمية منذ عصر قديم، ودائما بحامي التعايث السلمي وتعايش الإخوة و المحبة. ورذه الرؤيةتوجدفي طينها وطبيعتها من حيث 
في هذه الثعار ات و الإشار ات. وما أعظم هذه الفكرة وما أنبلها، وكم هي قريبة من رؤية المو اطنة العالمية. في الو اقع تحتضن أرض الهند في مهدها مواد المواطنة العالمية وهي تشكل ثاني أكبر دول بنسبة السكان وهي سكن لأكثر من سبعة عشر في المئة من إجمالي عدد السكان في العالم، فإذا جرب العدد الكبير في العالم منظور المواطنة العالمية ورجدوا أنفسهر مفتخرين بها، فمعنى ذلك أن منظور المواطنة العالمية قد حقق انجاز ا كبيرا جدير ا بالذكر. ولا شك في التاريخ أن الهند يعتبر من أقدم الحضارات العالمية ومنبعا لحضار ات أخرى في العالم وهو يلفت أنظار الباحثين إليه، وله أهمية كبرى على المستوى العالمي. منذ أن ولدت في هذا البلد الذي

لقب بجنة الأرض وتر عرعت بين و اديه الجميل، و أمضيت أيام طفولتي وصباي بين خضراوته ومياهه العذبة ،و جربت
حق الملائكة وتكرمها إكراما5tithi Devo Bhava" رؤية المواطنة العالمية. و هو يتفاعل مع كل شخص مهما اختلفت ثقافته ولغته ودينه، ويحترم التنوع بل يفتخر به ويجعل شعاره " الوحدة في التنوع" فما كانت تسبب في خلق الصراعات و الجدال و الاصطدام أصبحت قوة وسببا للعيش السلمي في الهند وهذا بسبب الانفتاح و القبول لكل ثقافة ودين .فخرج الهند من منظور وطني بحت إلى وطني و اسع، ويمتهن مهار ات المشاركة المدنية و الفعالية السياسية، و التعاطف الثقافي، واحترام التنوع، والقدرة على التوفيق و التحكيم بين الصر اعات والتوصل إلى توافق في الآراء بطريقة المفاوضات والحوار والتسوية القضائية أو غيرها من الوسائل السلمية، بما في ذللك الوكالات والتنظيمات الإقليمية.انظر كيف رسخت جذور المواطنة العالمية

\footnotetext{
${ }^{5}$ Taittiriya Upanishad, Shikshavalli I.11.2
} 
وهذا كله أصبح ممكنا بسبب الرؤية الهندية التي وجدناها من أبائنا وأجدادنا كتر اث غال، والتي تعلمنا السلم و الأخوة والأمن والاحترام والافتخار بالثقافة و الانفتاح للثقافة الأخرى و إكرام عادات

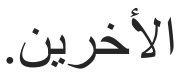

و هذا كانت تجربتي في القرى والأحياء وفي ميادين الألعاب و محافل الأعياد، وقويت هذه التجربة عندما التحقت بجامعة شهيرة هندية تعرف بوسعة فكر هاو عمق معرفتها وهي في الحق كانت نوافق أحلام رئيس الوزراء الهندي الأول جواهر لال نهروالذي يقول:" من مسؤولية الجامعة أن تقف بجانب الإنسانية وأن تسامح وأن تبحث عن الواقع والصدق وأن تقف لمقاصد عليا، وإن كانت الجامعات تحصل ما ذكر فيما أعلاه فهي تخدم لصالح المجتمع والدول."66 ولا شك إ ذ ذرعيت هذه الجامعة قد نالت تماما من أهدافها

${ }^{6}$ Mahatma Gandhi, $7^{\text {th }}$ April, Indian opinion.
مرارا وتكرارا مثال التعايش السلمي و التصالح والتسامح بين الثعوب المختلفة في دينها ولونها وعاداتها وثقافتها مع وحدة التعاطف الإنساني. لا يكون أي منظر أحسن وأجمل من هذا المنظر عندما يشارك كل القرية في الفرحة والسرور كما هي تشارك في وقت الأحزان والألام والأسى. و إني رأيت هذا المنظر بقريب إذ كان المسلم يرقص على مطراب الهندوس وهما مختلفان في الديانة و الثقافة.وكنت أتعجب كثيرا إذا أسمع أنباء الصراع بين المجتمعين في أنحاء العالم، وكنت لا لاء أتنقن بها. وكيف لا أتعجب بينما علمني بلادي وقريتي التعايش السلمي و التسامح و المشاركة، فإن احتفال العيد لا يتم بدون مشاركة أصدقائنا الهندوسية، و واحتفال الألوان لا تتم بدون مشّاركة المسلمين. نعم هذا البلا الهندي بيت للمجتمعات الكثيرة. كنا نشأنا و أكلنا كثير ا في كل بيوت ولا نعلم هل هو مسلم أو هندوسي. 
و إن هذه الجامعة جامعة جواهر لال نهرو التي تعتبر مركز ا مهما للتعليم العالي لا في الهند فقط بل في العالم، وهي التي جعلت أفق فكري واسعة ووفرتني فرصة اللقاء مع الأصدقاء ذوات الثعوب والقيم والأديان المختلفة من أقصى الهند و أدناه، ومن موطني أنحاء العالم من أوربا إلي الأمريكان،

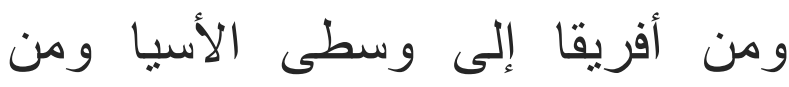
أستراليا إلى مناطق أخرى في العالم. و هذه الجامعة جامعة سكنية يعيش في

حرمها الأبناء والبنات كمجتمع واحد ممتعة بالحرية الكاملة، وهنالك ترى المواطنة العالمية في كل مكان من موقف البأسات إلى المقاهي ومن الكليات إلى الفنادق والمطاعم ومن الحجرات إلى ميادين الجامعة. بعد زيارة هذه الجامعة تخطر في ذهنك صور ا رائعة عندما ترى أفردا من أجناس مختلفة جالسين أمامك متحدثين بلغة مختلفة متلبسين بلباس بلدهم، ومفتخرين
وهي الجامعة التي مكنت لي أن أكتب هذه السطور. . وهذه الجامعة ليست جامعة فقط بل هي صورة جميلة وعائلة صغيرة للثقافات و اللغات العالمية. تعتبر كلية لغتها وثقافتها من أكبر كلية الجامعة ومن أكبر كلية اللغة والثقافة في أسيا أيضا. و هنا أحس أهية تعدد اللغات إذ رعيت الطلاب والأساتذة للغات العديدة و الثقافات المختلفة قد جمعت تحت مظلة واحدة، ويمكن لي هناك أن أرى ألوان صور الحضارات العالمية بقرب وهي هعطي إحساسا جميلا في القلب وقرارا في العين، وهنا أتمنى وأفكر كم كان العالم أجمل لو كان كهذه الجامعة. وكم نحن سعداء وفرحين لو كنا نلتقي مع الحضار ات الأخرى و أناس مناطق العالم بدون أي صعوبة الحدود والتأتثيرات و الجائزة.والله كان العالم أجمل وأحسن لو يلتقي مع القيم والعادات الأخرى في أكنافه و أنحائه، ألا نرى صورة ممتعة لملتقى الأبحر، ما هي بهاؤها وحسنها. 
الجنس أو اللغة أوالدين ولا تفريق بين الرجال والنساء، و إنها فعلت كثير ا في محو الأمية والإفلاس وإزالة الفوارق الجنسية و العصبية.و إنها دائما تؤكد على التتمية المستدامة ،وعقدت البرامج المختلفة لتبادل الأراء والأفكار و و حاربت عدم المساو اة وعززت التضامن و الرعاية البئية.

وتستحق الأمم المتحدة كل التقدير و الثكر و الثناء على ما أدت دور ها في مجال التعليم. و إنها في الحقيقة تمثل خطوة مهمة موفقة لتحقيق هدف المواطنة العالمية في وقت قريب. وقد قال الأمين العام للأمم المتحدة موكدا على أهمية التعليم: التعليم هو القوة الرئيسية للتنمية البشرية، و إنه يفتح باب العمل، ويقوم بمبدء المساواة، ويساعد في إحسان صحة الأمومة، ويقلل من معدلات أموات الصبيان. و التعليم يؤفر المرء المعرفة والممارسة والقيم التي تجب لبناء عالم أفضل. وقال أيضا: إن
بثقافتهم، قد تشعر بعد رؤية هذا المنظر البهيج أن العالم كله قد تجمع في جامعة واحدة تحت مظلة التعليه. و العنصر المهم الذي جعل هذا كله ممكنا هو التعليم. وينتج من ذللك أن التعليم له دور مهم جدا في محو الفوارق العصبية الجنسية و الطائفية و استيصال العصبيات الدينية واللونية من جذورها وتكوين المواطنة العالمية المبنية على أساس الحرية والعدالة الاجتماعية و السياسية و الاقتصادية، وفي جعل بناء عالم أفضل يمتلي بالفرح و الأمن و السلام. دور الأمم المتحدة في التقريب بين الأمم والشعوب: و لا شك أن الأمم المتحدة قد لعبت ولا نز ال تلعب دورا ملموسا منذ فجر ها الأول في التعاون الدولي في الميادين الاقتصادية والاجتماعية والثقافية و التعليمية والصحية وفي الإعانة على تحقيق حقوق الإنسان والحريات الأساسية للناس كافة بلا تمبيزبينهم في 

المؤسسات بسبب العرق أو اللون أو

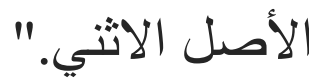

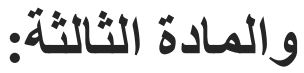

"تبذل جهود خاصة لمنع التمبيز بسبب العرق أو اللون أو الأصل الاثني لا سيما في ميادين الحقوق المدنية، ونيل

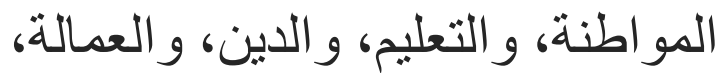

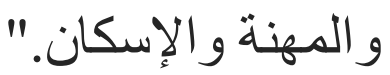
والمادة الحادية عشر :

"تقوم كل دولة بتعزيز احترام

ومر اعاة حقوق الإنسان و الحريات الأساسية وفقا لميثاق الأمم المتحدة الأنسان وبالالتز ام التام الدقيق لأحكام هذا لالحا

الإعلان و الإعلان العالمي لحقوق الإن الإن الإن الإنسان و إعلان منح الاستقلاعل للبلدان و الثعوب المستمرة. وتثص المادة الثالثة عشر:

"لكل فرد حرية التنقل واختيار محل إقامته داخل حدود كل دولة. ويحق

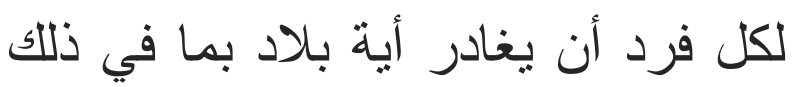
بلده كما بحق له العودة إلبه". 8

7 حقوق الإنسان: مجموعة صكوك دولية، المجلد الأول، الأمم المتحدة، نيويورك،

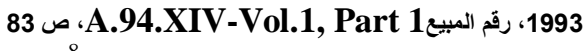

${ }^{8}$ www.un.org
المبادرة التعليمية لها ثلاث مرجحات: الأول: وضع كل طفل في المعهد العلمي و الثاني: تحسين نوعية التعليم و الثالث: تعزيز رؤية المواطنة العالمية. ومن هنا واضح أن التعليم يعد حجر ا أساسيا نحو بناء الوطن العالمي. والإعلان العالمي لحقوق الإنسان للجمعية العامة للأمم المتحدة هو أول خطوة إلى تحقيق هذذا الهدف حيث:

\section{تنص المادة الأولى من الإعلان:}

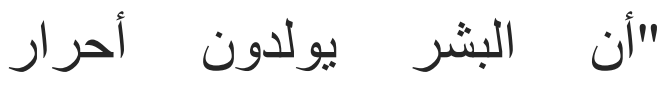
و منساوين في الكر امة و الحقوق، و أن من حق كل إنسان أن يتمتع بجميع الحقوق و الحريات المقررة في الإعلان، دون أب لي تمبيز، لا سيما بسبب العرق أو اللون أو لوك الأصل القومي.

\section{وتتص المادة الثانية:}

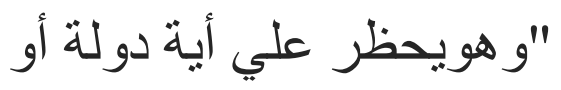
مؤسسة أو جماعة أو أي فرد إجر اء أب دي دولي تمييز كان، في ميدان حقوق الإنسان و الحريات الأساسية، في معاملة الأشخاص أو جماعات الأشخاص أو فئل 
وأحسن ما قال شاعر الثرق العلامة إقبال: "بلاد الله بلادنا وليس لنا بلد مختص." وما أحسن سقر اط في إجابته عن وطنه: " وطني ليس إثنيا ولكن

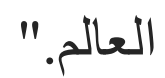

ولا بد أن تؤكد المواطنة العالمية على حل هذه القضايا المشتركة التي تهم الجميع، مثلا عى قمع أعمال العدوان وإزالة الفقر ومحو الأمية، ونشر الأمن والسلام ومبادئ المساواة بين الجميع و الحرية وتوفير الفرص للجميع والاعتناء بتعليم الأطفال خاصة في الدول المتخلفة، وتبادل الأراء و التو اصل وتقليل الأمراض و الحد من تفتي المخدرات و المحذورات وغيرها من الأمور التي تحتاج إلى العناية العالمية. و لا بد أن تحتذر من التدخل في الثُؤون الدولية التي تقدر عليه الدولة في حلها ومعالجنها، ولا بد الاحتراز من حماية ثقافة ولغة خاصة كما يجب الاحتر از من الإغفال عن حضارة ولسان أخر. وبهذا
هذه التصريحات تشكل أهية كبرى في هذا القرن، قرن التطور وقرن الأمال وقرن التحديات و الذي يملأ بالقضايا العديدة، من أهمها قضايا البيئة و الحرب و الإرهاب و الكو ارث الطبيعية و الإنسانية و الجريمة السيير انية و التجارة و السياسة وقضايا الأسلحة النووية وقضايا الصحة و الغذاء و التعليم وقضايا الحقوق الإنسانية الأخرى. و وهذه التصريحات لها أهية كبيرة للى لهيك المحرومين والمتخلفين وطردى و المجروحين،و الهاربين من الأوطان و البيوت وخاصة الأطفال و النساء التي تنظر إليها بنظر الأمال. تتطلب هذه القضايا المساعي المشتركة من كل العالم لأن دولة واحدة أو مجتمعا واحدا لا يستطيع أن يحلها هذه لهن المشاكل كالجبال،فالحاجة ماسة إلى جعل عولمة هذه القضايا التي يشارك فيها الجميع و المواطنة العالمية خطوة جميلة تكسب لهم جوا ملائما يتتفسون فيه الصعداء. 
العالمية" عالمي، وفي العالم توجد ثقافات ولغات كثيرة، و هي مانعة من الحوار وتبادل الأراء، ولذا يجب تو اجد الأفر اد و الماهرين في لغات عديدة في أن و احد و هم يلِعبون دور الجسور بين بحور الثقافات و اللغات. فيلعب تعدد اللغات دورا أساسيا في جمع الثعوب و المجتمعات على رصيف و احد وجر ها إلى اجتماع الطاولة المستديرة. وتستطيع الأفر اد الذين يتكيفون بأسلحة اللغات المتعددة ، ولهم يد لا تنكر في هذا المجال، وبإمكانهم ربط العالم بأجمعه بخيط واحد إنساني، وبإمكانهم تحويل هذا العالم إلى بستان ملئ بالأز هار المفتحة الخلابة التي تجد ماءها من بئر عذب، و الذي يقع على شاطئ الأنهار الجارية و على و اديها أثجار طويلة تتغرد العصافير على أغصانهاوتلعب الأطفال تحت ظلالها الو ارفة من كل أنحاء العالم. و هذا كله ممكن من تعدد اللغات و المو اطنة العالمبة.
الطريق يمكن نجاح الفكرة العالمية على نطاق أوسع، كما بمكن إزالة المخاوف التي ربما تسيطر على أفكار عامة الناس حول ظاهرة المواطنة العالمية. ومن أهم المخاوف هي: المواطنة العالمية هي وسيلة هيمنة ثقافة أو لغة خاصة على ثقافات أخرى، أو هي هيمنة الدول المتقدمة على الحياة الاجتماعية و السياسية والاقتصادية على المستوى العالمي، أو هي فكرة لاستغلال الدول المتطورة والمتخلفة. فيجب من توضيح الفرق بين العولمة و العالمية و المحلية، كما يجب تحديد الأهداف و المقاصد التي تسعى الأمم المتحدة لإنجاز ها.

الخلاصة أهمية تعدد اللغات ومما تجدر بالذكر أن تحقيق هذه الأهداف ليست سهلة و إنما هي تقتضي الجهود المكثفة و المتو اصلة بكل صدق و إخلاص، و لأن موضوع "المو اطنة 
